\title{
Indicadores Climáticos e a Produtividade de Soja no Rio Grande do Sul
}

\author{
Diogo Alessandro Arsego ${ }^{1}$ (D), Simone Erotildes Teleginski Ferraz ${ }^{2}$, Nereu Augusto Streck ${ }^{3}$, \\ Andréa de Oliveira Cardoso ${ }^{4}$, Alencar Junior Zanon ${ }^{3}$ \\ ${ }^{1}$ Centro de Previsão de Tempo e Estudos Climáticos, Instituto Nacional de Pesquisas Espaciais \\ Cachoeira Paulista, SP, Brasil. \\ ${ }^{2}$ Departamento de Física, Universidade Federal de Santa Maria, Santa Maria, RS, Brasil. \\ ${ }^{3}$ Departamento de Fitotecnia, Universidade Federal de Santa Maria, Santa Maria, RS, Brasil. \\ ${ }^{4}$ Universidade Federal do ABC, Santo André, SP, Brasil.
}

Recebido em: 1 de Junho de 2018 - Aceito em: 15 de Outubro de 2018

\begin{abstract}
Resumo
O objetivo deste trabalho foi identificar padrões de teleconexão que influenciem na variabilidade da produtividade de soja no Rio Grande do Sul de forma a encontrar potenciais variáveis preditoras da produtividade agrícola no Estado. Foram utilizados dados de produtividade de soja de 87 municípios fornecidos pelo Instituto Brasileiro de Geografia e Estatística. Estas séries foram reunidas em três grupos homogêneos de produtividade mediante análise de agrupamento. Correlações defasadas entre índices climáticos associados a padrões de teleconexão e a produtividade média da soja de cada um dos três grupos evidenciaram a importância de cada índice para a cultura no Rio Grande do Sul. Dentre os padrões de teleconexão, os que apresentaram correlações mais significativas, confiança superior a 90\%, e persistentes com a produtividade de soja foram a Oscilação Ártica (negativa entre outubro e dezembro), Oscilação do Atlântico Norte (negativa entre outubro e janeiro) e anomalias de Temperatura da Superfície do Mar no Oceano Atlântico, entre $20^{\circ} \mathrm{S}-30^{\circ} \mathrm{S}$ e $20^{\circ} \mathrm{O}-40^{\circ} \mathrm{O}$, (positivas entre dezembro e março). Estes índices também apresentaram elevada correlação com a precipitação compreendida entre dezembro a março para cada grupo homogêneo evidenciando a importância do seu monitoramento para o planejamento da safra de soja no Rio Grande do Sul.
\end{abstract}

Palavras-chave: indicadores climáticos, correlação, Glycine max L, variabilidade interanual.

\section{Climatic indicators and soybean yield in Rio Grande do Sul}

\begin{abstract}
The main of this work was to identify teleconnection patterns that influence soybean yield variability in Rio Grande do Sul in order to find potential predictors of agricultural yield in the State. Soybean yield data from 87 municipalities provided by the Brazilian Institute of Geography and Statistics were used. These series were grouped into three homogeneous groups of yield by cluster analysis. Lag correlations between climatic indexes associated with teleconnection patterns and mean soybean yield of each of the three groups evidenced the importance of each index for the crop in Rio Grande do Sul. Among the teleconnection patterns, the ones that presented most significant correlations, confidence higher than $90 \%$, and persistent with soybean yield were the Arctic Oscillation (negative between October and December), North Atlantic Oscillation (negative between October and January) and Sea Surface Temperature anomalies in the Atlantic Ocean, between $20^{\circ} \mathrm{S}-30^{\circ} \mathrm{S}$ and $20^{\circ} \mathrm{O}-40^{\circ} \mathrm{W}$, (positive between December and March). These indices also showed a high correlation with the precipitation between December and March for each homogeneous group evidencing the importance of their monitoring for the planning of the soybean farming in Rio Grande do Sul.
\end{abstract}

Keywords: climate index, Glycine $\max L$, correlation, interannual variability.

Autor de correspondência: Diogo Alessandro Arsego, diogo.arsego@gmail.com. 


\section{Introdução}

A soja (Glycine max L.) é uma cultura agrícola chave para a segurança alimentar global por ser uma fonte de proteína e energia para a nutrição humana e animal, respondendo por $56 \%$ da produção das oleaginosas no mundo (USDA, 2017). O Brasil é o segundo maior produtor de soja, com $31 \%$ da produção mundial, uma área cultivada de 34 milhões de hectares e uma produtividade média de $2,9 \mathrm{Mg} / \mathrm{ha}$ (IBGE, 2017). O Rio Grande do Sul (RS) é o terceiro Estado entre os produtores de soja no Brasil, com produção média de 5 anos (2012-2016) de $12 \mathrm{Mt}$ e produtividade média de $2,5 \mathrm{Mg} / \mathrm{ha}$ (IBGE, 2017). A maior parte da soja no RS é cultivada em regime de sequeiro (Sentelhas et al., 2015), sendo assim, a quantidade e distribuição da precipitação durante janeiro e março, torna-se o principal fator limitante à produtividade de grãos (Berlato e Fontana, 1999; Ferreira, 2006; Zanon et al., 2016).

Embora a região da América do Sul onde se localiza o RS seja caracterizada por um regime climático de precipitações bem distribuídas ao longo do ano e com totais pluviométricos elevados (Rao e Hada, 1990; Reboita et al., 2010), episódios de estiagem são recorrentes, sendo o principal fator que provoca quedas de produtividade agrícola nas culturas de sequeiro e prejuízos para a economia do Estado (Lazzari, 2006; Sentelhas et al., 2015). Quebras d safras de soja podem acarretar grandes prejuízos socioeconômicos ao país, o que foi verificado nos anos agrícolas de 2004/2005, 2008/2009 e 2011/2012, devido à ocorrência de estiagens que se estenderam sobre toda a Região Sul, sendo o último ano o mais severo, no qual ocorreu o fenômeno La Niña e maiores perdas nas lavouras do RS, cerca de 43\% (Leivas et al., 2014). Assim, a compreensão dos padrões meteorológicos responsáveis pela variabilidade interanual da precipitação sobre o Estado é fundamental para tomadas de decisões sobre as melhores épocas de semeadura de forma a minimizar possíveis efeitos da variabilidade do clima na produtividade das culturas agrícolas (Zanon et al., 2016; Zanon et al., 2018).

A passagem de frentes frias sobre o RS é o principal mecanismo causador de precipitação na região e que contribui para que o padrão climatológico de precipitação seja pouco variado ao longo do ano, sendo maior a frequência destes eventos na primavera (Andrade, 2007; Cardozo et al., 2015). Assim, fenômenos climáticos que possam afetar a atividade de sistemas transientes, influenciam o regime de chuvas nesta região, podendo impactar as atividades agrícolas.

Estudos demonstram que o fenômeno acoplado El Niño - Oscilação Sul (ENOS) apresenta sinal significativo sobre o sudeste da América do Sul e suas influências na variabilidade da precipitação e da produtividade agrícola da soja no RS já foram estudadas (Grimm et al., 1998;
Berlato e Fontana, 1999). Apesar de outros padrões de teleconexão como a Oscilação Decadal do Pacífico (Mantua et al., 1997), Oscilação Antártica (Cavalcanti e Ambrizzi, 2009) e anomalias de Temperatura da Superfície do Mar no Oceano Atlântico (Cataldi et al., 2010; Santos e Diniz, 2014) estarem intimamente relacionados a variabilidade no regime de precipitação sobre o RS, o impacto destes padrões de teleconexão na produtividade de soja no Estado ainda não foi estudado.

Mesmo com a evolução tecnológica da agricultura e das diferentes técnicas de manejo (rotação de culturas, cobertura permanente do solo com material vegetal), que buscam minimizar os impactos das variações dos estresses hídricos no rendimento das culturas, períodos prolongados de estiagens ou precipitações excessivas podem impactar fortemente uma safra agrícola (Radin et al., 2017; Zanon et al., 2018). Sendo a soja altamente dependente da precipitação registrada durante o seu ciclo de desenvolvimento, a identificação de mecanismos de teleconexão que impactam a precipitação e, consequentemente a produtividade de soja, faz-se necessária para aprimorar as estimativas de safra, fornecendo mais subsídios no momento em que é realizado o planejamento das lavouras de soja. Neste contexto, o objetivo foi identificar índices climáticos associados a padrões de teleconexão que afetem os fatores agrometeorológicos e possuam maior influência na variabilidade da produtividade de soja no RS.

\section{Materiais e Métodos}

Nesta análise foram utilizados dados de produtividade de soja dos 497 municípios do RS, fornecidos pelo Instituto Brasileiro de Geografia e Estatística (IBGE), entre os anos de 1974 a 2013, sendo excluído desta análise o ano de 1983 por não constar na base de dados. Para eliminar dados espúrios ou séries de municípios de pouca representatividade para a cultura foram adotados dois filtros: eliminação dos municípios com dados faltantes e/ou repetidos por três anos consecutivos e remoção de séries que apresentassem correlação inferior a 0,7 com a produtividade média do Estado.

Séries históricas de produtividade apresentam tendência de crescimento ao longo dos anos por conta de melhoramentos nas técnicas de cultivo e aumento do potencial de produtividade das cultivares de soja. Para estudos da influência da variabilidade climática, esta tendência necessita ser removida (Bannayan et al., 2010). A tendência tecnológica foi removida das séries de produtividade de soja dos 87 municípios restantes por meio de uma regressão linear simples (Eq. 1) na qual assume-se que a tendência linear da série é resultado unicamente de incrementos na tecnologia (Wenjiao et al., 2013).

$$
Y_{c i}=Y_{i}-Y\left(X_{i}\right)+Y\left(X_{f}\right)
$$


sendo $Y_{c i}$ - produtividade corrigida do ano $i ; Y_{i}$ - produtividade original do ano $i ; Y\left(X_{i}\right)$ - produtividade do ano $i$ estimada pelo modelo de regressão; $Y\left(X_{f}\right)$ - produtividade do último ano da série estimada por meio da segunda regressão linear para que a série coincida no último valor.

Para verificar quais municípios apresentavam características semelhantes quanto à produtividade, estabeleceram-se três grupos homogêneos com base na análise de agrupamento. As séries restantes foram divididas em municípios de alta, média e baixa produtividade durante $\mathrm{o}$ período de estudo. A medida de distância utilizada foi a distância Euclidiana (Wilks, 2006) e para o agrupamento foi utilizado o critério hierárquico proposto por Ward (1963).

Com a distribuição dos municípios produtores em grupos homogêneos, foram obtidas três séries médias de produtividade de soja. Estas séries médias foram então correlacionadas com os seguintes indicadores climáticos disponibilizados pela página do "National Oceanic and Atmospheric Administration - NOAA": Anomalia de TSM na região do Niño 3.4 (NINO - Trenberth, 1997); Índice de Oscilação Sul (SOI - Trenberth et al., 1984); Oscilação do Atlântico Norte (NAO - Rogers, 1985); Padrão do Pacífico América Norte (PNA - Wallace e Gutzler, 1981); Oscilação Decadal do Pacífico (PDO - Mantua et al., 1997); Modo Meridional do Atlântico (AMM - Servain, 1991); Oscilação Ártica (AO - Thompson e Wallace, 1998) e Oscilação Antártica (AAO - Thompson e Wallace, 2000). Estes índices passaram por uma seleção prévia sendo retidos apenas os que apresentaram correlações significativas com a produtividade de soja.

Além dos indicadores acima listados, foram adicionados dois índices adicionais: ATSM1 e ATSM2. O índice ATSM1 é referente à região do Atlântico Sul delimitada entre as latitudes de $20^{\circ} \mathrm{S}$ e $30^{\circ} \mathrm{S}$ e as longitudes $20^{\circ} \mathrm{O}$ e $40^{\circ} \mathrm{O}$. Anomalias de Temperatura da Superfície do Mar (TSM) nessa região possuem correlação positiva com a precipitação no RS, principalmente, entre outubro e janeiro (Santos e Diniz, 2014). O índice ATSM2 está relacionado à região compreendida entre $33^{\circ} \mathrm{S}$ e $43^{\circ} \mathrm{S}$ e $48^{\circ} \mathrm{O}$ e $63^{\circ} \mathrm{O}$. Anomalias positivas (negativas) de TSM na região de encontro entre as Correntes do Brasil e das Malvinas alteram o regime de chuvas e favorecem precipitações abaixo (acima) da média no Sul (Sudeste) (Cataldi et al., 2010).

Estes índices foram criados por meio da normalização dos dados observados da seguinte forma (Eq. 2):

$$
I P=\frac{\left(x_{i j}-\bar{x}_{i}^{-}\right)}{\sigma_{i}}
$$

sendo IP o índice padronizado referente a cada área de TSM; $x_{i j}$ o valor observado no mês $i$ e ano $j$, no caso da TSM o valor corresponde a média da TSM na região de estudo; $\bar{x}_{i}$ a média de todas as observações do mês $i$ e $\sigma_{i} \mathrm{o}$ desvio padrão das observações no mês $i$.

Foram realizadas correlações defasadas entre os índices climáticos associados a cada padrão de teleconexão e a produtividade média da soja de cada um dos três grupos. Assumiu-se abril como mês referente a colheita por ser o período em que é colhida a maior parte da soja no RS (Fontana et al., 2001). As correlações foram analisadas com base nos indicadores de maio a dezembro do ano anterior e de janeiro a abril do ano de colheita. Desta forma, a primeira correlação é tomada entre o índice climático referente ao mês de maio do ano anterior e a produtividade do ano subsequente (lag 11), a segunda entre o índice do mês de junho do ano anterior e a produtividade do ano seguinte até a última correlação entre o índice do mês de abril do ano referente à colheita e o valor de produtividade deste ano (lag 0 ).

A significância das correlações foi estimada com base no conceito de hipótese nula. A hipótese nula $\left(\mathrm{H}_{0}\right)$ assume que um dado resultado estatístico foi obtido por conta do acaso e não devido a um efeito real que cause o resultado. Ao se trabalhar com uma hipótese para explicar um dado fenômeno, deve-se considerar a possibilidade de ao menos uma hipótese concorrente a ela, a hipótese alternativa $\left(\mathrm{H}_{1}\right)$ (Murray et al., 2013).

Neste estudo, considerou-se o resultado empírico no qual a correlação entre os índices listados e as três séries médias de produtividade é perfeita. Como hipótese nula, considerou-se que este resultado é pura coincidência e, como hipótese alternativa considerou-se o contrário, que a correlação é significante. Assumiu-se o limiar do valor de probabilidade abaixo do qual a hipótese nula é rejeitada de $10 \%(\mathrm{p}=0,10)$, ou seja, um nível de confiança de $90 \%$. Se a probabilidade do evento for menor que $10 \%$, rejeita-se a hipótese nula; caso a probabilidade for maior que $10 \%$, não se pode rejeitar a hipótese nula.

Com base em um teste bilateral pela distribuição de $t$ de Student (Fisher e Yates, 1974) ao nível de 0,10 (10\%) com n-2 graus de liberdade, sendo $n$ o número de casos (39 anos de dados), e $t_{0}=\frac{r \sqrt{n-2}}{\sqrt{1-r^{2}}}$, os valores de t0 calculado e r(gl) são: 1,6879 e 0,267, respectivamente. Para o índice referente à $\mathrm{AAO}$, por se tratar de uma série menor (1979 a 2013, $n=34)$, os valores de $\mathrm{t}(\mathrm{gl})$ e r(gl) são diferentes: 1,6938 e 0,287 , respectivamente.

Se $r>r(g l)$ ou $r<-r(g l)$, rejeita-se $\mathrm{H}_{0}$ e aceita-se $\mathrm{H}_{1}$, ou seja, a correlação é significativa e há correlação entre as variáveis consideradas. Neste estudo, foram consideradas as correlações significativas ao nível de $10 \%$.

Após a obtenção de grupos homogêneos de produtividade, séries municipais de precipitação mensal provenientes da Agência Nacional de Águas foram selecionadas de forma a obter um perfil histórico médio para cada grupo homogêneo. Desta forma, foi possível realizar análises de correlação entre a precipitação compreendida entre 
dezembro e março e as séries de produtividade de forma semelhante a apresentada em Berlato e Fontanta (1999). Também foi estabelecida uma relação entre os índices climáticos com impacto na produtividade com as séries de precipitação de cada grupo homogêneo.

\section{Resultados e Discussão}

A distribuição dos municípios em grupos homogêneos de produtividade de soja permitiu delimitar regiões do RS com diferentes padrões de produtividade média. Desse agrupamento, ficou delimitado que os municípios com maior produtividade de soja estão localizados no nordeste do Estado (Grupo 1 - G1), os municípios com média produtividade estão distribuídos entre o norte e centro do Estado (Grupo 2 - G2) e os municípios localizados no noroeste do RS apresentam uma menor produtividade (Grupo 3 - G3) (Figs. 1 e 2).

De acordo com o zoneamento agroclimático da soja no RS, o G1 está localizado em uma região na qual a produtividade de soja é limitada pela baixa disponibilidade térmica o que, segundo Melo et al., (2004), poderia ser superado pelo uso de cultivares precoces ou atraso na data de semeadura (início de novembro). Também na região onde predominam os municípios do G1, o risco de queda de produtividade por déficit hídrico no solo é menor (Cunha et al., 2001). O G2 encontra-se na região preferencial para a semeadura de soja e sua menor produtividade, em relação ao G1, pode ser explicada pelo maior

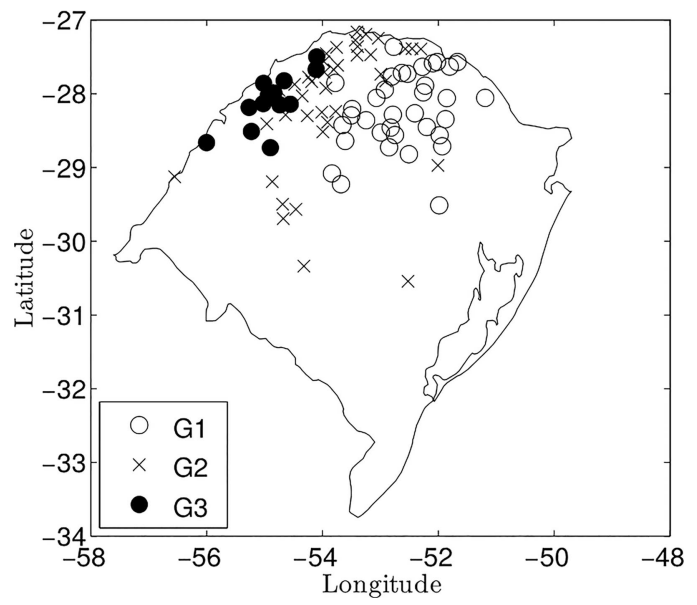

Figura 1. - Mapa do Rio Grande do Sul com as regiões homogêneas de produtividade de soja (Grupos G1, G2 e G3) resultante da análise de agrupamento.

risco de deficiência hídrica em comparação com o G1 (Cunha et al., 2001). Por outro lado, o G3 (baixa produtividade), apesar de estar localizado entre a área preferencial e tolerada, está localizado em uma região onde as perdas de produtividade estão relacionadas ao déficit hídrico, sendo a irrigação uma prática de manejo recomendada para aumentar a produtividade, porém ainda incipiente (Melo et al., 2004).

A produtividade de soja está intimamente ligada a precipitação acumulada quando a cultura encontra-se na

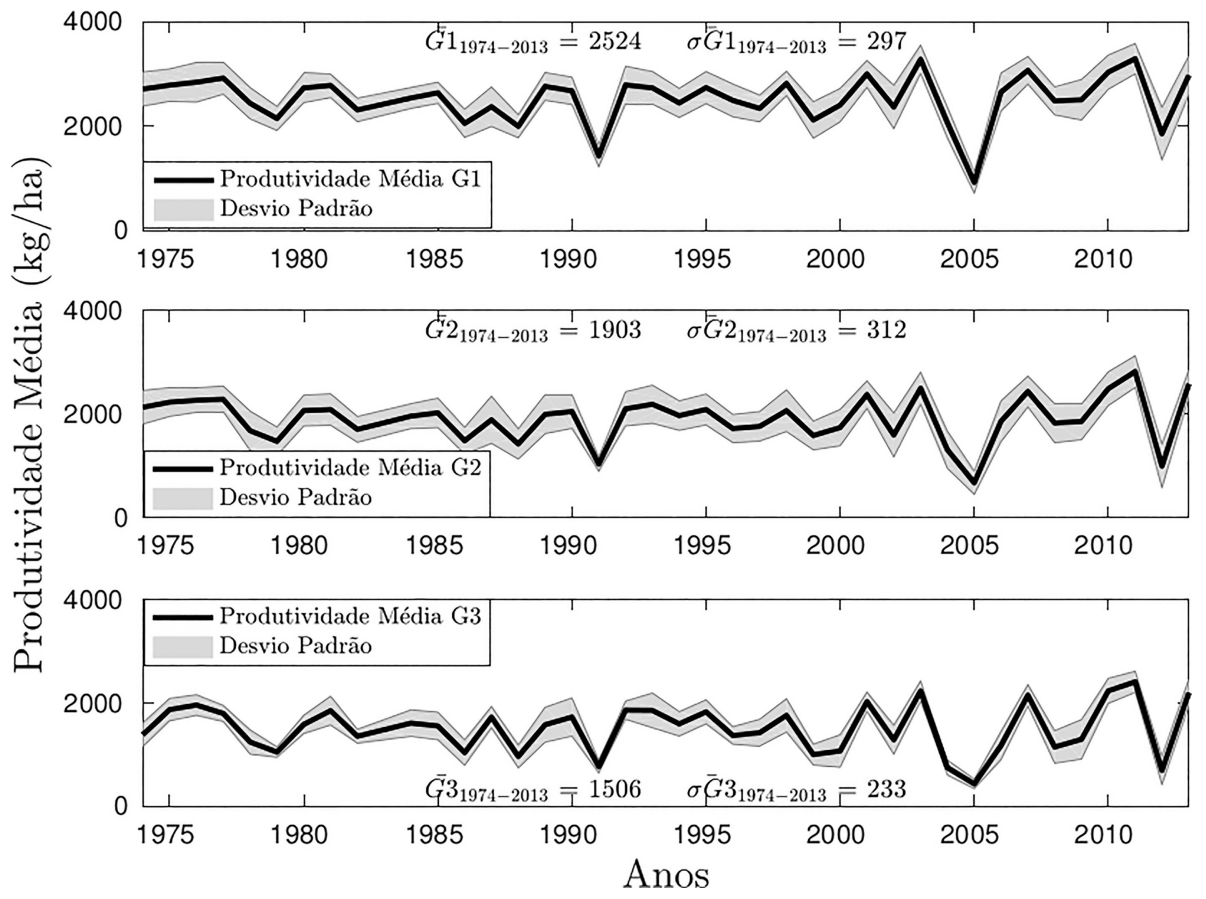

Figura 2. - Perfis médios de produtividade de soja para os três grupos homogêneos resultantes da análise de agrupamento: G1 (painel superior), G2 (painel central) e G3 (painel inferior). A área sombreada corresponde ao desvio padrão de cada série. Na Figura também estão inseridos os valores médios da produtividade e o desvio padrão médio para todo o período de estudo. 
fase reprodutiva, entre os meses de dezembro e março (Tabela 1), resultado muito semelhante ao apresentado nos estudo prévio de Berlato e Fontana (1999) e Zanon et al. (2016). Os índices climáticos associados a padrões de teleconexão utilizados neste estudo têm influência na variabilidade da precipitação no RS e, consequentemente, pressupõe-se que estão relacionados indiretamente com a produtividade de soja.

$\mathrm{Na}$ Tabela 2 estão listados os índices climáticos que apresentaram correlação a um nível de confiança de, no mínimo, 90\% com um dos grupos de produtividade de soja. Nas correlações baseadas nos indicadores climáticos para o mês de maio do ano anterior a colheita, o índice ATSM2 apresentou para o G2 os melhores resultados com correlação positiva com $90 \%$ de confiança. Como aplicação prática deste resultado, dada a lenta variação da TSM e levando em conta que a maior parte da soja no RS é semeada a partir de outubro, tal correlação para o G2 poderia servir como potencial indicativo da produtividade de soja na região central do Estado com considerável antecedência.

Para junho, nota-se a influência da AMM que apresenta uma correlação negativa significativa a $90 \%$ com o Grupo 3. Em estudo sobre a variabilidade na vazão de vários rios sobre o Brasil, Capozzoli et al., (2017) demonstraram uma correlação negativa da AMM com a vazão, variável meteorológica ligada diretamente a precipitação, sobre a Bacia do Uruguai para defasagens temporais de até 4 meses com o índice precedendo a vazão. Assim como para a vazão, os resultados aqui encontrados evidenciam que anomalias positivas (negativas) de TSM no Atlântico tropical norte acompanhadas de anomalias negativas (positivas) de TSM no Atlântico tropical sul durante o mês de junho indicariam uma potencial queda (elevação) da produtividade de soja no noroeste do RS.

Tabela 1 - Correlação entre a precipitação compreendida nos períodos DEZ/JAN/FEV/MAR, DEZ/JAN/FEV e JAN/FEV/MAR com a produtividade média de soja para os Grupos 1 (G1), 2 (G2) e 3 (G3).

\begin{tabular}{lccc}
\hline & Grupo 1 & Grupo 2 & Grupo 3 \\
\hline DEZ/JAN/FEV/MAR & 0,76 & 0,70 & 0,73 \\
DEZ/JAN/FEV & 0,67 & 0,66 & 0,67 \\
JAN/FEV/MAR & 0,72 & 0,61 & 0,70 \\
\hline
\end{tabular}

Tabela 2 - Índices climáticos mensais com correlação com a produtividade média de soja no Rio Grande do Sul dos três grupos homogêneos de produtividade (G1, G2 e G3) a um nível de confiança de $90 \%$ e o sinal das correlações. Os índices e defasagens não listados não apresentaram correlação ao nível de confiança estabelecido.

\begin{tabular}{|c|c|c|c|}
\hline & Grupo 1 & Grupo 2 & Grupo 3 \\
\hline & NS & ATSM2 maio $(+)$ & NS \\
\hline & NS & NS & AMM junho (-) \\
\hline & $\mathrm{AO}$ agosto $(+)$ & $\mathrm{AO}$ agosto $(+)$ & $\mathrm{AO}$ agosto $(+)$ \\
\hline & PNA agosto (-) & PNA agosto (-) & PNA agosto (-) \\
\hline & AO outubro (-) & AO outubro (-) & AO outubro (-) \\
\hline & NAO outubro (-) & NAO outubro (-) & NAO outubro (-) \\
\hline & AO novembro (-) & AO novembro (-) & NS \\
\hline & NAO novembro (-) & NAO novembro (-) & NS \\
\hline \multirow[t]{11}{*}{ ÍNDICES } & NS & NS & PDO novembro $(+)$ \\
\hline & AO dezembro (-) & AO dezembro (-) & AO dezembro (-) \\
\hline & NAO dezembro (-) & NAO dezembro (-) & NAO dezembro (-) \\
\hline & ATSM1 dezembro $(+)$ & ATSM1 dezembro $(+)$ & ATSM1 dezembro $(+)$ \\
\hline & AAO janeiro (-) & AAO janeiro (-) & AAO janeiro (-) \\
\hline & NAO janeiro (-) & NAO janeiro (-) & NAO janeiro (-) \\
\hline & NS & PNA janeiro $(+)$ & PNA janeiro $(+)$ \\
\hline & ATSM1 janeiro (+) & ATSM1 janeiro (+) & ATSM1 janeiro (+) \\
\hline & ATSM1 fevereiro $(+)$ & ATSM1 fevereiro $(+)$ & ATSM1 fevereiro $(+)$ \\
\hline & ATSM1 março (+) & ATSM1 março (+) & ATSM1 março (+) \\
\hline & NS & $\mathrm{AO}$ abril $(+)$ & $\mathrm{AO}$ abril $(+)$ \\
\hline
\end{tabular}


Nos meses de julho e setembro, os índices climáticos utilizados no presente estudo não apresentaram correlação significativa com nenhum dos grupos homogêneos de produtividade de soja. Para agosto, padrões de teleconexão que possuem influência mais direta no clima do Hemisfério Norte apresentam sinal na produtividade de soja no RS. A relação destes índices do Hemisfério Norte com a produtividade agrícola no Hemisfério Sul pode sugerir influências inter-hemisféricas, mas merecem uma análise futura mais detalhada. A Oscilação Ártica (AO) possui correlação positiva e com $90 \%$ de confiança para os três grupos. O PNA também possui relação com a produtividade de soja nos três grupos, porém com sinal negativo.

A influência de padrões do Hemisfério Norte também é evidenciada entre outubro e dezembro e, destaca-se a persistência da correlação negativa entre os índices referentes à $\mathrm{AO}$ e NAO com a produtividade para os três grupos, sendo mais forte no mês de outubro para os dois índices. Como a maior parte da soja no RS é semeada entre outubro e novembro (Fontana et al., 2001), e por possuir uma persistência significativa da correlação nos meses subsequentes, os índices climáticos do mês de outubro referentes à $\mathrm{AO}$ e NAO também se mostram como bons indicadores da produtividade de soja no RS.

Os índices climáticos a partir do mês de novembro perdem seu caráter prognóstico do ponto de vista de prevenções para eventuais quebras de safra, tendo em vista que a soja já foi semeada em todo o Estado. Por isso, correlações significativas a partir de novembro servem como diagnóstico de como a cultura da soja poderá se desenvolver e crescer nos meses seguintes. Em novembro, a PDO apresenta correlação positiva a $90 \%$ com o G3. Sabe-se que na América do Sul a PDO atua construtivamente (destrutivamente) ao fenômeno ENOS quando na mesma fase (fase oposta) (Kayano e Andreoli, 1998). Embora as correlações referentes às anomalias de TSM relacionadas ao El Niño não apresentem significância estatística, de forma geral, o G3 apresenta os maiores valores, reforçando a importância do índice referente a PDO no mês de novembro para este grupo.

Em dezembro, além da AO e da NAO acima citadas, os índices relacionados a TSM da área ATSM1 passam a apresentar sinal significativo, pois esta área apresenta impacto relevante na precipitação de verão sobre o RS (Santos e Diniz, 2014). As correlações positivas relacionadas a ATSM1 persistem nos meses de janeiro, fevereiro e março, período do ano agrícola da soja em que a precipitação é fundamental para garantir a produtividade da cultura, pois neste período a maioria das lavouras estão na fase reprodutiva (floração e enchimento de grãos), a mais sensível ao déficit hídrico (Zanon et al,. 2016). No mês de janeiro ainda há uma correlação negativa significativa a $90 \%$ com a NAO e positiva a $90 \%$ tanto para o G2 quanto para o G3 com a PNA. Destaca-se também a correlação negativa com a AAO no mês de janeiro. A AAO está intimamente ligada a atividade frontogenética em latitudes médias (Cavalcanti e Ambrizi, 2009) e, em sua fase negativa (positiva), está associada a anomalias positivas (negativas) de precipitação sobre o RS (Vasconsellos 2012).

No mês de março, os índices referentes a ATSM2 apresentam correlação negativa ao nível de confiança de $90 \%$ com a produtividade de soja para o G3. Anomalias positivas de TSM na região da ATSM2 estão relacionadas a um período de diminuição da precipitação sobre parte do Sul do Brasil (Cataldi et al., 2010) e, por ser um mês onde a demanda por água vinda da precipitação ainda é alta pela cultura que está em enchimento de grãos, o G3 apresenta uma queda de produtividade nestas condições.

Por fim, no mês de abril, quando a maior parte das lavouras de soja no RS são colhidas, nota-se uma correlação positiva (90\%) da $\mathrm{AO}$ com os grupos 2 e 3 . Nota-se que a $\mathrm{AO}$ apresenta correlações significativas e negativas entre os meses de outubro a janeiro e, à medida que se aproxima a época de colheita, há modificação em seu sinal e alcança sua máxima correlação positiva no mês de abril.

Destaca-se a baixa correlação da componente oceânica (NINO) e atmosférica (IOS) do ENOS. Tal situação pode ser explicada por conta da maior influência do ENOS na precipitação da primavera (Lopes et al., 2007), período em que a maior parte da soja ainda está sendo semeada. Além disso, a separação por fases do evento (não apresentada) demonstra que uma correlação linear positiva é observada em casos de El Niño (El Niños favorecem a cultura da soja no RS), mas em casos de neutralidade e La Niña, a influência do fenômeno na produtividade de soja não fica clara (anos de La Niña e neutralidade podem ser bons ou ruins para a cultura da soja no RS). De fato, Alberto et al. (2006) já haviam destacado que episódios de neutralidade apresentam maior risco de déficit hídrico e consequente queda de produtividade se comparados a eventos de La Niña.

Na Tabela 3, estão os índices climáticos com correlação com a precipitação, compreendida entre dezembro e março, a um nível de confiança superior a $90 \%$. Os resultados confirmam a possibilidade que os índices climáticos exercem influência na variabilidade da precipitação sobre o RS e, de forma indireta, impactam também nos índices de produtividade de soja registrados no Estado. Assim como para a correlação com a produtividade de soja, a relação encontrada entre alguns índices climáticos e a precipitação não possui um mecanismo físico conhecido que explique tais resultados (AO, PNA e NAO), embora os resultados aqui apresentados evidenciem que há uma influência na precipitação e, posteriormente, na produtividade de soja no RS.

As Fig. 3 e 4 apresentam a dispersão entre os índices climáticos do mês de agosto referentes a $\mathrm{AO}$ e ao PNA associados a desvios de produtividade e precipitação para os três grupos homogêneos. Estes índices foram escolhidos por serem, dentre os índices com correlação 
Tabela 3 - Índices climáticos mensais com correlação com a precipitação compreendida entre dezembro e março nos três grupos homogêneos de produtividade (G1, G2 e G3) no Rio Grande do Sul a um nível de confiança de $90 \%$ e o sinal das correlações. Os índices e defasagens não listados não apresentaram correlação ao nível de confiança estabelecido.

\begin{tabular}{|c|c|c|c|}
\hline & Grupo 1 & Grupo 2 & Grupo 3 \\
\hline \multirow{19}{*}{ ÍNDICES } & ATSM2 maio $(+)$ & NS & \\
\hline & NS & NS & AMM junho (-) \\
\hline & $\mathrm{AO}$ agosto $(+)$ & $\mathrm{AO}$ agosto $(+)$ & $\mathrm{AO}$ agosto $(+)$ \\
\hline & PNA agosto (-) & PNA agosto (-) & PNA agosto (-) \\
\hline & AO outubro (-) & AO outubro (-) & AO outubro (-) \\
\hline & NAO outubro (-) & NAO outubro (-) & NAO outubro (-) \\
\hline & AO novembro (-) & AO novembro (-) & NS \\
\hline & NAO novembro (-) & NAO novembro (-) & NS \\
\hline & NS & NS & PDO novembro $(+)$ \\
\hline & AO dezembro (-) & AO dezembro (-) & AO dezembro (-) \\
\hline & NAO dezembro (-) & NAO dezembro (-) & NAO dezembro (-) \\
\hline & ATSM1 dezembro $(+)$ & ATSM1 dezembro $(+)$ & ATSM1 dezembro $(+)$ \\
\hline & AAO janeiro (-) & AAO janeiro (-) & AAO janeiro (-) \\
\hline & NAO janeiro (-) & NAO janeiro (-) & NAO janeiro (-) \\
\hline & NS & PNA janeiro (+) & PNA janeiro (+) \\
\hline & ATSM1 janeiro (+) & ATSM1 janeiro $(+)$ & ATSM1 janeiro $(+)$ \\
\hline & ATSM1 fevereiro $(+)$ & ATSM1 fevereiro $(+)$ & ATSM1 fevereiro $(+)$ \\
\hline & ATSM1 março (+) & ATSM1 março (+) & ATSM1 março (+) \\
\hline & & AO abril (+) & $\mathrm{AO}$ abril $(+)$ \\
\hline
\end{tabular}
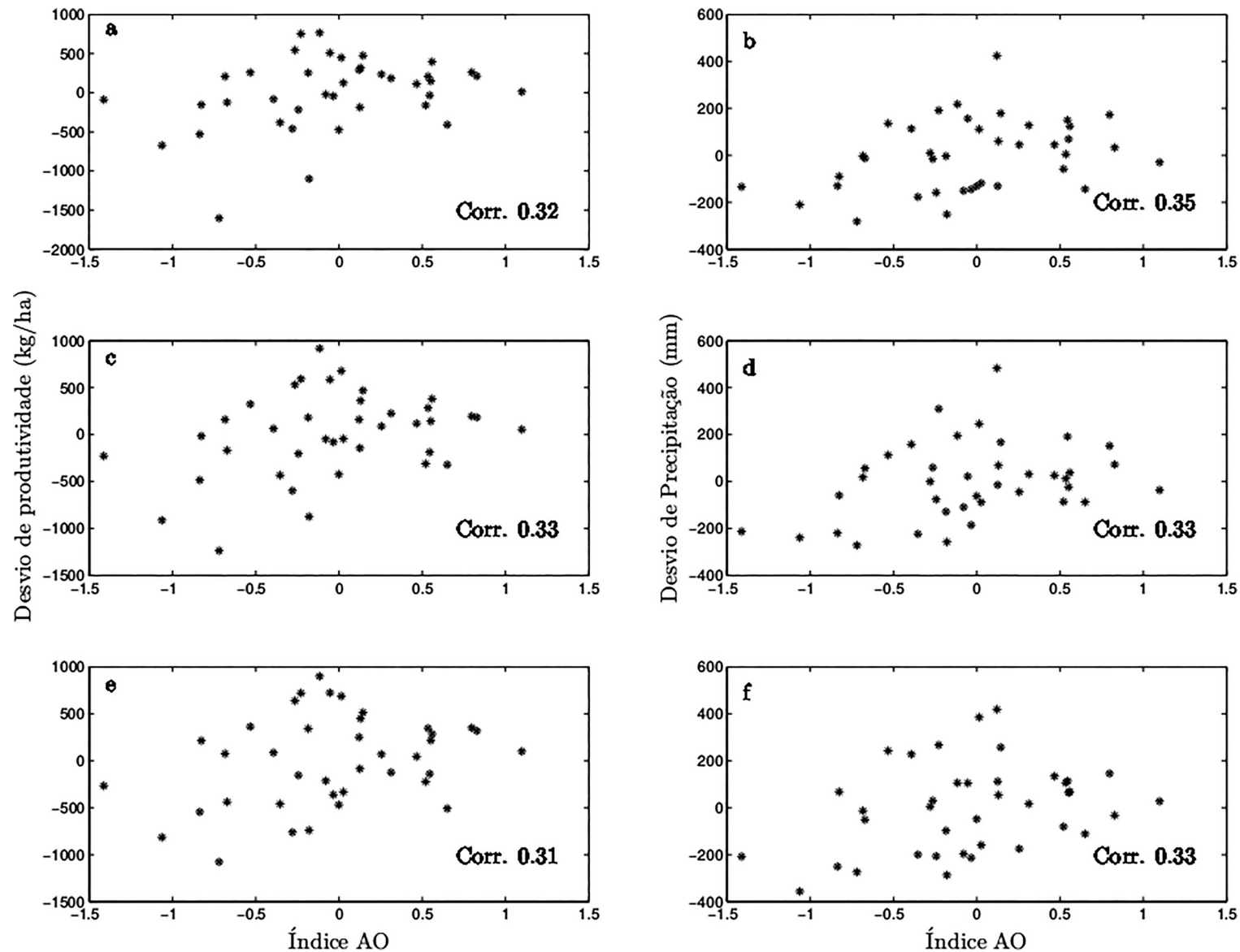

Figura 3. - Relação entre o índice de agosto do $\mathrm{AO}$ e desvios de produtividade de soja (a, c e e) e de precipitação entre dezembro e março (b, d e f) para os grupos homogêneos G1 (a e b), G2 (c e d) e G3 (e e f) no Rio Grande do Sul. No gráfico consta também o valor da correlação linear entre as variáveis. 

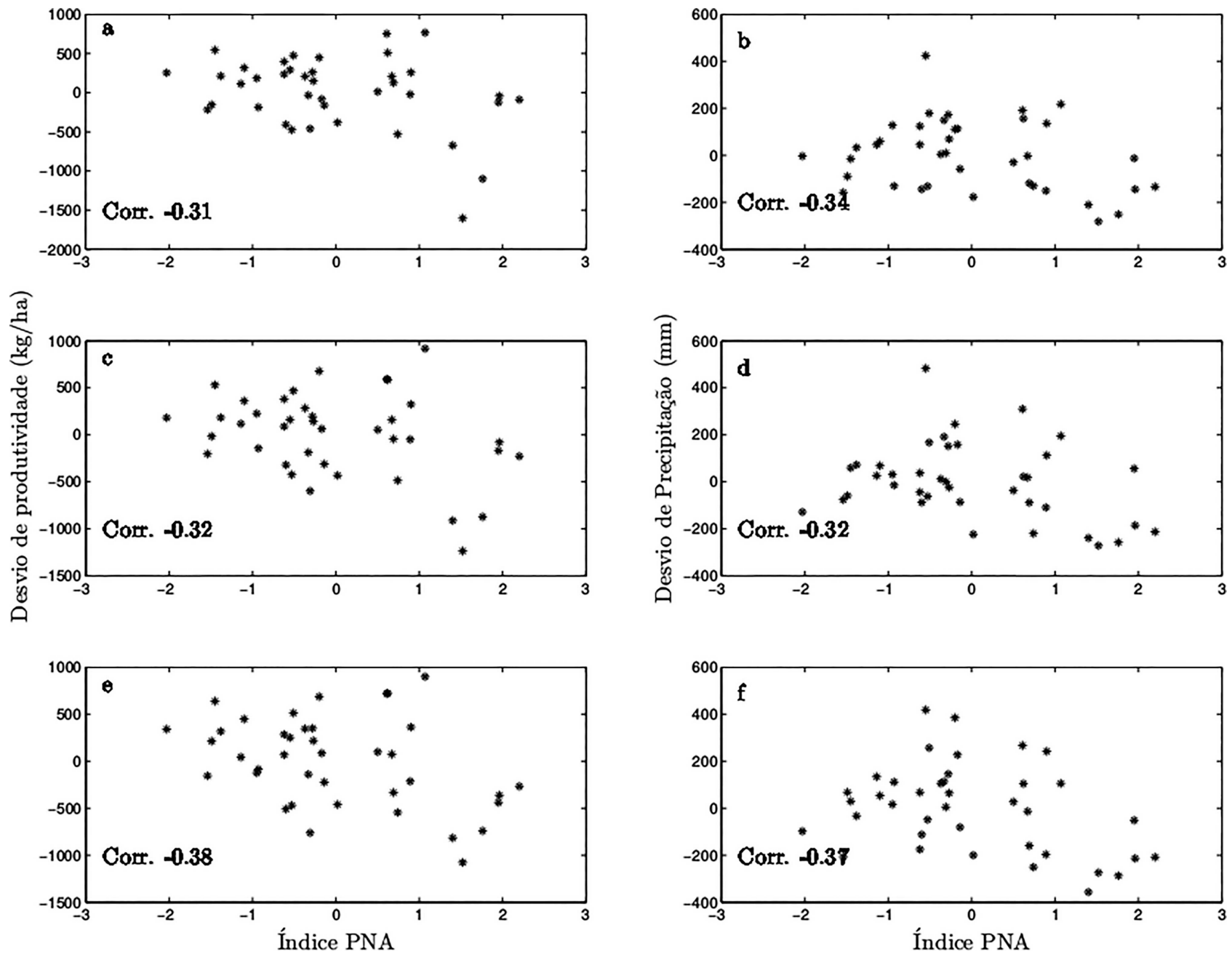

Figura 4. - Relação entre o índice de agosto da PNA e desvios de produtividade de soja (a, c e e) e de precipitação entre dezembro e março (b, d e f) para os grupos homogêneos G1 (a e b), G2 (c e d) e G3 (e e f) no Rio Grande do Sul. No gráfico consta também o valor da correlação linear entre as variáveis.

significativa, os que apresentaram maior defasagem para os três grupos. Para a AO (Fig. 3), nota-se uma correlação positiva com nível de confiança de $90 \%(|r|>0,287)$ entre o índice referente ao mês de agosto e os desvios de produtividade para os três grupos homogêneos (Figs. 3a, 3c e 3e). A mesma correlação positiva é observada entre o índice de agosto referente a $\mathrm{AO}$ e os desvios de precipitação no G1 (Fig. 3b), G2 (Fig. 3d) e G3 (Fig. 3f). A análise de dispersão referente ao índice de agosto relacionado ao PNA apresenta correlações negativas entre o índice e a produtividade de soja nos três grupos (Figs. 4a, $4 c$ e 4e) resultante da influência negativa deste padrão de teleconexão na precipitação registrada entre dezembro e março para os três grupos (Figs. 4b, 4d e 4f).

A compreensão dos padrões de teleconexão responsáveis pela variabilidade da produtividade de soja no RS pode ser uma ferramenta prática para auxiliar consultores técnicos e os produtores para um melhor planejamento da safra e na mitigação de eventuais perdas de produtividade. Os resultados aqui apresentados indicam que índices climáticos podem ser usados para entender e explicar a variabilidade da produtividade de soja no Rio Grande do Sul, o que ainda não havia sido reportado na literatura.

De uma forma geral, nota-se uma maior influência de padrões associados ao Hemisfério Norte para defasagens mais longas e do Hemisfério Sul para defasagens mais curtas. Desta forma, indicadores mais remotos apresentam-se como previsores potenciais da safra de soja no RS enquanto padrões do Hemisfério Sul poderiam ser utilizados no acompanhamento da safra após o período de semeadura da cultura.

\section{Conclusões}

Os índices relacionados à Oscilação Ártica (AO), ao Pacífico América Norte e à Oscilação do Atlântico Norte (NAO) possuem sinal significativo para os três grupos homogêneos de produtividade de soja do Rio Grande do Sul. Além destes, as anomalias de Temperaturas de Superfície do Mar na área entre $20^{\circ} \mathrm{S}-30^{\circ} \mathrm{S}$ e $20^{\circ} \mathrm{O}-40^{\circ} \mathrm{O}$ 
também apresentam significativa correlação, porém em um período no qual a semeadura da soja, normalmente, já está finalizada no RS, caracterizando-se assim, como índice que poderia auxiliar em possíveis correções de estimativas da produtividade.

Sugere-se a inclusão de tais índices em previsões e discussões do planejamento da safra de forma a contribuir para melhores estimativas da produtividade da soja no Rio Grande do Sul.

\section{Referências}

ALBERTO, C.M.; STRECK, N.A.; HELDWEIN, A.B.; BURIOL, G.A.; MEDEIROS, S.L.P. Água no solo e rendimento do trigo, soja e milho associados ao El Niño Oscilação Sul. Pesquisa Agropecuária Brasileira, v. 41, n. 7, p. 1067-1075, 2006.

ANDRADE, K. Climatologia e comportamento dos sistemas frontais sobre a América do Sul. 2007. 187 f. Dissertação (Mestrado em Meteorologia) - Instituto Nacional de Pesquisas Espaciais, 2007.

BANNAYAN, M.; SANJANI, S.; ALIZADEH, A.; LOTFABADI, S.S.; MOHAMADIAN, A. Association between climate indices, aridity index and rainfed crop yield in northeast of Iran. Fild Crop Research, v. 118, p. 105-14, 2010.

BERLATO, M.A.; FONTANA, D.C. Variabilidade interanual da precipitação pluvial e rendimento da soja no Rio Grande do Sul. Revista Brasileira de Agrometeorologia, v. 7, n. 1, p. 119-125, 1999.

CAPOZZOLI, C.; CARDOSO, A. de O.; FERRAZ, S.E.T. Padrões de variabilidade de vazão de rios brasileiros e associação com índices climáticos. Revista Brasileira de Meteorologia, v. 32, n. 2, p. 243-254, 2017.

CARDOZO, A.B.; CUSTÓDIO, I.S.; REBOITA, M.S.; GARCIA, S.R. Climatologia de frentes frias na América do Sul e sua relação com o Modo Anular Sul. Revista Brasileira de Climatologia, v. 17, p. 9-29, 2015.

CATALDI, M.; ASSAD,L.P. de F.; JUNIOR, A.R.T.; ALVES, J. L.D. Estudo da influência das anomalias da TSM do Atlântico Sul Extratropical na Região da Confluência BrasilMalvinas no regime hidrometeorológico de verão no Sul e Sudeste do Brasil. Revista Brasileira de Meteorologia, v. 25, n. 4, p. 513-524, 2010.

CAVALCANTI, I.F. de A.; AMBRIZZI, T. Teleconexões e suas influências no Brasil. In: CAVALCANTI, I.F. de A.; FERREIRA, N.J.; SILVA, M.G. A.J. da; DIAS, M.A.F. da S. (Ed.). Tempo e Clima no Brasil. [S.I.]: Oficina de Textos, 2009. P. 317-336.

CUNHA, G.R. da; BARNI, N.A.; HAAS, J.C; MALUF, J.R.T.; MATZENAUER, R.; PASINATO, A. Zoneamento agrícola e época de semeadura para soja no Rio Grande do Sul. Revista Brasileira de Agrometeorologia, v. 9, n. 3, p. 446-459, 2001.

FERREIRA, D. B. Relação entre a variabilidade da precipitação e a produtividade agrícola de soja e milho nas Regiões Sul e Sudeste do Brasil. 2006. 125 f. Dissertação (Mestrado em Meteorologia) - Instituto Nacional de Pesquisas Espaciais, São José dos Campos, 2006.
FISHER, R.A.; YATES. F. Statistical Tables for Biological, Agricultural and Medicinal Research, 6. ed. London, England: Longman, 1974.

FONTANA, D.C.; BERLATO, M.A.; LAUSCHNER, M.H.; MELLO, T.W. de. Modelo de estimativa de rendimento de soja no Estado do Rio Grande do Sul. Pesquisa Agropecuária Brasileira, v. 36, n. 3, p. 399-403, 2001.

IBGE (2017). Produção agrícola municipal. URL: https://sidra. ibge.gov.br/Tabela/1612.

GRIMM, A.M.; FERRAZ, S.E.T.; GOMES, J. Precipitation Anomalies in Southern Brazil Associated with El Niño and La Niña Events. International Journal of Climatology, v. 27, p. 531-540, 1998.

HOOGENBOOM, G. Contribution of agrometeorology to the simulation of crop production and its applications. Agricultural and Forest Meteorology, v. 103, p. 137-157, 2000.

KAYANO, M.T.; ANDREOLI, R.T. Relations of South American Summer rainfall interannual variations with the Pacific Decadal Oscillation. Journal of Climate, v. 11, p. 28632880, 1998.

LAZZARI, M.R. Agropecuária gaúcha em 2005: estiagem, câmbio e crise. Indicadores Econômicos FEE, v. 33, n. 4, p. 4150, 2006.

LEIVAS, J.F.; ANDRADE, R.G.; VICTORIA, D. de C.; TORRESAN, F.E.; VICENTE, L.E.; TEIXEIRA, A.H. de C.; BOLFE, E.L.; BARROS, T.R. de. Avaliação do índice de vegetação padronizado no monitoramento indicativo de estiagens em períodos críticos da soja no Sul do Brasil. Revista Brasileira de Cartografia, n. 66/5, p. 1145-1155, 2014.

LOPES, F.Z.; DINIZ, G.B.; MARQUES, J.R. Relação entre o Multivariate Enso Index (MEI) e a TSM das regiões dos niños com a precipitação em regiões homogêneas do Estado do Rio Grande do Sul. Anuário do Instituto de Geociências, v. 30, p. 11-22, 2007

MANTUA, N.J.; HARE, S.R.; ZHANG, Y; WALLACE, J.M.; FRANCIS, R.C. A Pacific Interdecadal Climate Oscillation with Impacts on Salmon Production. Bulletin of the American Meteorological Society, v. 78, n. 6, p. 10691079, 1997.

MELO, R.W. de; FONTANA, D.C.; BERLATO, M. A. Indicadores de produção de soja no Rio Grande do Sul comparados ao zoneamento agrícola. Pesquisa Agropecuária Brasileira, v. 39, n. 12, p. 1167-1175, 2004.

SPIEGEL, M.R.; SCHILlER, J.J.; SRINIVASAN, R.A., Probabilidade e Estatística, Coleção Schaum, $3^{\text {a }}$ Edição, Editora Bookman, 2013

RADIN, B.; SCHONHOFEN, A.; TAZZO, I.F. Impacto da quantidade e freqüência de chuva no rendimento da soja. Agrometeoros, v. 25, n. 1, p. 19-26, 2017.

RAO, G. V.; HADA, K. Characteristics of rainfall over Brazil annual variations and connections with the Southern Oscillation. Theoretical and Applied Climatology, v. 42, p. 8191, 1990.

REBOITA, M.S.; GAN, M.A.; ROCHA, R.M. da; AMBRIZZI, T. Regimes de precipitação na América do Sul: uma revisão bibliográfica. Revista Brasileira de Meteorologia, v. 25, n.2, p. 185-204, 2010. 
ROGERS, J.C. Atmospheric circulation changes associated with the warming over the Northern North Atlantic in the 1920s. Journal of Climate Applied Meteorology, v. 24, n. 12, p. 1303-1310, 1985.

SANTOS, R.B.; DINIZ, G.B. Oceanic índices and their relations with the monthly precipitation in Rio Grande do Sul State, Brazil. Revista Brasileira de Geofísica, v. 32, p. 371-381, 2014.

SENTELHAS, P.C.; BATTISTI, R.; CÂMARA, G.S.; FARIAS, J.R.B.; HAMPF, A.C.; NENDEL, C. The soybean yield gap in Brazil - magnitude, causes and possible solution for sustainable production. Journal of Agricultural Science, v. 153, p. 1-18, 2015.

SERVAIN, J. Simple climatic indices for the tropical Atlantic Ocean and some applications. Journal of Geophysical Research, v. 96, n. C8, p. 15137-15146, 1991.

THRENBERTH, K.E. Signal versus noise in the Southern Oscillation. Monthly Weather Review, v. 112, n. 2, p. 326-332, 1984.

THENBERTH, K. E.; The definition of El Niño. Bulletin of American Meteorological Society, v. 78, n. 12, p. 27712777, 1997.

THOMPSON, D.W.J.; WALLACE, J.M. The Arctic Oscillation signature in the wintertime geopotential height and temperature fields. Geophysical Research Letters, v. 25, p. 1297-1300, 1998.

THOMPSON, D.W.J.; WALLACE, J.M. Annular Modes in the extratropical Circulation. Part 1: Month-tomonth variability. Journal of Climate, v. 13, p. 1000-1016, 2000.

USDA - UNITED STATES DEPARTMENT OF AGRICULTURE. Production, Supply and Distribution Online. Disponível na internet: http://www.usda.gov/wps/
portal/usda/usdahome?navid=DATA_STATISTICS. Acesso em 20/06/2017.

VASCONSELLOS, F.C. A Oscilação Antártica - mecanismos físicos e a relação com características atmosféricas sobre a América do Sul/oceanos adjacentes. 2012. $195 \mathrm{f}$. Tese (Doutorado em Meteorologia) - Instituto Nacional de Pesquisas Espaciais, São José dos Campos, 2012.

WALLACE, J.M.; GUTZLER, D.S. Teleconnections in the geopotential heigth field during the North Hemisphere winter. Monthly Weater Review, v. 109, n. 4, p. 784-812, 1981.

WARD, J.H. Hierarchical grouping to optimize an objective function. Journal of the American Statistical Association, v. 58, n. 301, p. 236-244, 1963.

WILKS, D.S. Statistical Methods in the Atmospheric Sciences. 2. ed. San Diego, California, United States of America: Academic Press, Elsevier, 2006. 630 p.

WENJIAO, S.; FULU, T.; ZHAO, Z. A review on statistical models for identifying climate contributions to crop yields. Journal of Geographical Sciences, v. 23, p. 567 - 576, 2013.

ZANON, A.J.; STRECK, N.A.; GRASSINI, P. Climate and management factors influence soybean yield potential in a subtropical environment. Agronomy Journal, v. 108, p. 1447-1454, 2016.

ZANON, A.J.; SILVA, M.R. ; TAGLIAPIETRA, E.L. ; CERA, J. C. ; BEXAIRA, K.P.; RICHTER, G.L. ; DUARTE, A.J. ; ROCHA, T.S.M.; WEBER, P.S.; STRECK, N.A. Ecofisiologia da Soja Visando Altas Produtividades. 1. ed. Santa Maria: Santa Maria: Palloti/SM, 2018. v. 1. 136p .

This is an Open Access article distributed under the terms of the Creative Commons Attribution License, which permits unrestricted use, distribution, and reproduction in any medium, provided the original work is properly cited. 